\title{
Diagnosing cystic fibrosis: what are we sweating about?
}

\author{
Nicholas J Simmonds, ${ }^{1}$ Andrew Bush ${ }^{2}$
}

Over the last few decades, the paradigm has shifted as cystic fibrosis (CF) is no longer a fatal disease of childhood and should be considered a chronic condition where survival into adulthood is expected. Median survival for current newborns is predicted to be at least 50 years and over $55 \%$ of patients in the UK are adults. ${ }^{1}$ Over these decades, our knowledge of the underlying pathophysiology has grown exponentially, from its original description in 1938 to the identification of the mutated gene (cystic fibrosis transmembrane conductance regulator, CFTR) in $1989 .^{2}$ This has driven the development of effective therapies and brought about an increased understanding of the wide spectrum of diseases that result from abnormal CFTR function. During this time, the humble sweat test has remained at the heart of the diagnostic algorithm, with only modest changes from the original pilocarpine iontophoresis technique first described by Gibson and Cooke over half a century ago. ${ }^{3}$ Abnormal sweat electrolytes result from dysfunctional or absent CFTR protein in the epithelial cells of sweat glands. ${ }^{4}$ CFTR is widely distributed throughout the body and has many functions, such as encoding a cAMP-activated chloride channel and regulating transepithelial ion movement-a phenomenon identified by the measurement of potential difference across the nasal mucosa and later used as the basis of the nasal potential difference (NPD) diagnostic test. ${ }^{5} 6$

With the identification of the mutated gene and extensive knowledge of the associated basic defect, why is there still such a debate about the criteria for diagnosis and why do different diagnostic algorithms exist? When is CF atypical or non-classic and why introduce yet more terms-'CFTR-related disorders' and 'CFTR-related metabolic syndrome'? Are 'Department of Cystic Fibrosis, Royal Brompton Hospital,
London, UK; ${ }^{2}$ National Heart and Lung Institute, Imperial
College, London, UK

Correspondence to Dr Nicholas J Simmonds, Department of Cystic Fibrosis, Royal Brompton Hospital, London SW3 6NP, UK; n.simmonds@rbht.nhs.uk these terms helpful or is this just overcomplicating matters? The study by Ooi et al is a valiant attempt at unravelling some of these issues by testing concordance between the two widely used algorithms of the American and European diagnostic guidelines for patients presenting with single-organ manifestations of disease. 7

The traditional approach of 'CF excluded', if the sweat chloride is $<60 \mathrm{mmol} / 1$ (or sodium $<70 \mathrm{mmol} / \mathrm{l}$ ), has been obsolete for many years and borne out by a greater understanding of the continuum of CFTR dysfunction and the tools available to measure it. Patients presenting in late childhood or adulthood initially gained attention in the 1980s as isolated case reports, but we now recognise an important cohort of patients who generally present late due to milder manifestations or single-organ involvement and, importantly, they are often pancreatic sufficient and therefore nutritionally replete. ${ }^{8}$ This is an important prognostic indicator as nutritional depletion from malabsorption is one of the strongest predictors of early presentation and more severe disease. ${ }^{9}$ When CFTR was first identified, it was thought that disease variability would be explained by different mutations, but the correlation of genotype with phenotype is generally poor, except for a few mutations that correlate with normal pancreatic exocrine function, for example, the $R 117 H$ mutation. ${ }^{10}$ However, the situation is made more complicated by the poor correlation of lung function severity with genotype, as two individuals with the same genotype may have very different lung function. Putative mechanisms to explain this variability include influences from airway infections (eg, chronic infection with Pseudomonas aeruginosa), and nutritional and environmental influences. Single nucleotide polymorphisms outside of CFTR ('gene modifiers') are also probably important. ${ }^{11}$ For these reasons, it is unhelpful to use the term 'mild' CF, as patients presenting late are still susceptible to these factors and are at risk of similar morbidity and rate of decline later in life as patients with a classic CF genotype/phenotype. ${ }^{12}$ Given this risk, American guidelines have tried to steer away from subdividing CF into classic/nonclassic (or atypical/typical), whereas European guidelines still recognise these terms in the context of the diagnosis 'CFTR dysfunction', which also includes 'CFTR-related disorders' ${ }^{13} 14$ The latter term has recently been defined by a joint European and American working group as 'a clinical entity associated with CFTR dysfunction that does not fulfil the diagnostic criteria for CF'. ${ }^{15}$

Despite CF being a monogenetic disease, molecular (genetic) testing is not always definitive, as failure to identify two CFTR mutations does not rule out the diagnosis. Over 1900 mutations have been identified thus far and the number is continuing to rise. ${ }^{16}$ First-line genotyping identifies the most common alleles in a given population (usually 29-50 mutations, which account for $85-90 \%$ of mutations), and whole CFTR scanning can be performed but this is expensive, time consuming and, with a detection rate of approximately $95 \%$, some patients will still be missed. This is complicated further by a limited understanding of the functional and clinical implications of very rare mutations and the impact of other genetic variations, such as single nucleotide polymorphisms. For this reason, after first-line genotyping, the American guidelines recommend only testing for the 23 mutations recognised as 'disease-causing' by the American College of Medical Genetics compared with the European guidelines, which recommend sequencing the whole gene and classifying patients according to the number of CFTR mutations identified. The latter approach will help us to better understand the relationship between phenotype, genotype and CFTR function, but, interestingly, in the study by Ooi et al, extended genotyping failed to improve the diagnostic yield by the American algorithm. An ambitious project is currently under way with the aim of fully categorising all CF mutations to enrich our understanding of the link between genotype and phenotype. ${ }^{17}$

Another important difference between the American and European guidelines is the thresholds for sweat chloride concentration. Both recognise that CF is very likely for a sweat chloride value $>60 \mathrm{mmol} / \mathrm{l}$, but the equivocal range is wider in the European algorithm (30-60 vs $40-60 \mathrm{mmol} / \mathrm{l}$ for patients older than 6 months)-recognition of a small but important cohort with low sweat chloride 
values and two disease-causing mutations (eg, compound heterozygote $3849+10 \mathrm{~kb}$ $\mathrm{C} \rightarrow \mathrm{T}){ }^{18}$ The American guidelines argue that this introduces too many false positives, as the upper limit of sweat chloride concentration in healthy controls has been determined as $39.5 \mathrm{mmol} / 1 .{ }^{19}$ The role of other physiological tests of CFTR function, such as NPD measurement, has been strongly debated for years. NPD is not fully incorporated into the American algorithm as it is regarded as an ancillary test, in contrast to the European algorithm, where it is an integral component used to provide definite evidence in uncertain cases. This discrepancy is justified in the American guidelines by the lack of clear reference values, validation studies and standardised technical protocols for NPD measurements. While diagnostic overlap and uncertainty can still occur with NPD, it has an important role in delineating the extent of CFTR function, thus moving individuals from one diagnostic classification to another, for example, CFTR-related disorder to 'CF' or 'unlikely CF' ( $17 \%$ of the total group in the study by Ooi et al were reclassified when NPD was introduced into the American algorithm). This is important because patients with an equivocal sweat chloride concentration with or without an abnormal NPD have been shown to differ phenotypically. ${ }^{20}$

Does the precise classification actually really matter? We would argue strongly that it does. Clearly, moving through either algorithm and being able to reach 'unlikely CF' is important as it effectively rules out the diagnosis and its implications. By contrast, the label of 'CF' may initially evoke negative connotations, but there can be relief at finally receiving a diagnosis for symptoms that have often persisted for years. Importantly, with the diagnosis comes access to specialist CF centres and the multidisciplinary $\mathrm{CF}$ team-a model of care that is associated with improved outcomes, ${ }^{21}$ including for patients diagnosed as having CF later in life. ${ }^{12}$ CF-specific drugs are well established and improve important clinical endpoints, such as $\mathrm{FEV}_{1}$ and frequency of CF lung attacks. ${ }^{22}$ Patients with unclassified disease or bronchiectasis from other causes will not routinely receive these drugs, and some drugs, for example, human recombinant DNase, may even have a deleterious effect. ${ }^{23}$ With the advent of mutation-specific therapies for CF and the real prospect of disease modification, confirmation of the diagnosis has never been more important. ${ }^{24}$
Where does this leave the term CFrelated disorders? The study by Ooi et al may be reassuring in the fact that concordance between the American and European guidelines was good to excellent with an overall agreement of $84.8 \%$, but differences were identified due to the lower limit of sweat chloride concentration and interpretations of genotypes. Importantly, NPD may not have improved concordance, but it did significantly shift individuals into different diagnostic classifications. A label of CFTR-related disorder goes some way to reduce the negative implications for individuals and their relatives of receiving the 'full' $\mathrm{CF}$ diagnosis (eg, psychological, reproductive, social, employment and insurance). However, as disease progression may still occur and prognostication is fraught with difficulty throughout the CF spectrum, confirmation of either diagnosis (CF or CFTR-related disorder) provides a framework by which to deliver specific care to ensure appropriate long-term surveillance and timely therapeutic interventions if necessary.

In spite of the issues discussed, it is important to recognise that for the majority of patients confirming the diagnosis of CF is straightforward, as they will fit the classic description of one or more phenotypic features with a sweat chloride value $>60 \mathrm{mmol} / \mathrm{l}$ and/or two CFTR mutations. Guidelines provide a structured, systematic and evidenced-based framework, but with this uncertainties may still exist, especially as our understanding of a complex disease like CF is evolving. Even now, some patients do not fit securely into a CF diagnostic category (they are termed 'inconclusive' by European guidelines)these patients may still be at risk of future complications and, therefore, should be followed-up carefully in the long term, at least until better diagnostic and prognostic biomarkers are identified. We agree with the concluding comment by Ooi et al, that the diagnostic algorithms should be regarded as guidelines, not dogma. It is vital we continue to characterise the disease throughout its spectrum to fully understand the interactions between mutated CFTR, its dysfunctional protein and the downstream clinical manifestations. With this we can furnish our knowledge, ameliorate uncertainty and work towards a robust unified diagnostic algorithm.

Contributors NJS wrote the first draft of the manuscript after discussion with $A B ; A B$ and NJS edited the subsequent drafts.

Competing interests None.
Patient consent Obtained.

Provenance and peer review Commissioned; internally peer reviewed.

Published Online First 17 April 2012

Thorax 2012:67:571-573

doi:10.1136/thoraxjnl-2012-201758

\section{REFERENCES}

1. Dodge JA, Lewis PA, Stanton M, et al. Cystic fibrosis mortality and survival in the UK: 1947-2003. Eur Respir J 2007:29:522-6.

2. Riordan JR, Rommens JM, Kerem B, et al. Identification of the cystic fibrosis gene: cloning and characterization of complementary DNA. Science 1989;245:1066-73

3. Gibson L, Cooke R. A test for concentration of electrolytes in sweat in cystic fibrosis of the pancreas utilizing pilocarpine by iontophoresis. Pediatrics 1959;23:545-9.

4. Quinton PM. Chloride impermeability in cystic fibrosis. Nature 1983;301:421-2.

5. Knowles M, Gatzy J, Boucher R. Increased bioelectric potential difference across respiratory epithelia in cystic fibrosis. N Engl J Med 1981;305:1489-95.

6. Alton EW, Currie D, Logan-Sinclair R, et al. Nasal potential difference: a clinical diagnostic test for cystic fibrosis. Eur Respir J 1990;3:922-6.

7. Ooi CY, Dupuis A, Ellis L, et al. Comparing the American and European diagnostic guidelines for cystic fibrosis: same disease, different language. Thorax 2012:67:618-24.

8. Nick JA, Rodman DM. Manifestations of cystic fibrosis diagnosed in adulthood. Curr Opin Pulm Med 2005;11:513-18.

9. Corey M, Farewell V. Determinants of mortality from cystic fibrosis in Canada, 1970-1989. Am J Epidemiol 1996;143:1007-17.

10. Anon. Correlation between genotype and phenotype in patients with cystic fibrosis. The Cystic Fibrosis Genotype-Phenotype Consortium. N Engl J Med 1993;329:1308-13

11. Drumm ML, Konstan MW, Schluchter MD, et al. Genetic modifiers of lung disease in cystic fibrosis. N Engl J Med 2005;353:1443-53.

12. Nick JA, Chacon CS, Brayshaw SJ, et al. Effects of gender and age at diagnosis on disease progression in long-term survivors of cystic fibrosis. Am J Respir Crit Care Med 2010;182:614-26.

13. De Boeck K, Wilschanski M, Castellani C, et al. Cystic fibrosis: terminology and diagnostic algorithms. Thorax 2006:61:627-35.

14. Farrell PM, Rosenstein BJ, White TB, et al. Guidelines for diagnosis of cystic fibrosis in newborns through older adults: cystic Fibrosis Foundation consensus report. J Pediatr 2008;153:S4-S14.

15. Bombieri C, Claustres M, De BK, et al. Recommendations for the classification of diseases as CFTR-related disorders. J Cyst Fibros 2011;10 (Suppl 2):S86-102.

16. Cystic Fibrosis Mutation Database. 2011. http://www genet.sickkids.on.ca/cftr/ (accessed 8 Mar 2012).

17. Clinical and functional translation of CFTR website. 2011. http://cftr2.com (accessed 8 Mar 2012).

18. Augarten A, Kerem BS, Yahav Y, et al. Mild cystic fibrosis and normal or borderline sweat test in patients with the $3849+10 \mathrm{~kb} \mathrm{C} \rightarrow \mathrm{T}$ mutation. Lancet 1993:342:25-6.

19. Mishra A, Greaves R, Smith K, et al. Diagnosis of cystic fibrosis by sweat testing: age-specific reference intervals. J Pediatr 2008;153:758-63.

20. Goubau C, Wilschanski M, Skalicka V, et al. Phenotypic characterisation of patients with intermediate sweat chloride values: towards validation of the European diagnostic algorithm for cystic fibrosis. Thorax 2009;64:683-91. 
21. Mahadeva R, Webb K, Westerbeek RC, et al. Clinical outcome in relation to care in centres specialising in cystic fibrosis: cross sectional study. BMJ 1998;316:1771-5.

22. Fuchs HJ, Borowitz DS, Christiansen DH, et al. Effect of aerosolized recombinant human DNase on exacerbations of respiratory symptoms and on pulmonary function in patients with cystic fibrosis. The Pulmozyme Study Group. N Engl J Med 1994;331:637-42.

23. O'Donnell AE, Barker AF, llowite JS, et al. Treatment of idiopathic bronchiectasis with aerosolized recombinant human DNase I. rhDNase Study Group. Chest 1998;113:1329-34.

24. Ramsey BW, Davies J, McElvaney NG, et al. A CFTR potentiator in patients with cystic fibrosis and the G551D mutation. N Engl J Med 2011; 365:1663-72

\section{Journal club}

\section{Cytisine improves smoking cessation}

In this single-centre, randomised, double blind, placebo-controlled trial, the efficacy and safety of cytisine as an aid in smoking cessation was compared with placebo. Three hundred and seventy participants were randomly assigned to either the active drug or placebo in an equal ratio for 25 days. Participants in both groups received a minimal amount of counselling during the study.

The primary outcome measure was sustained biochemically-verified smoking abstinence for 12 months after the end of treatment. Secondary outcomes were sustained abstinence for the first 6 months and point prevalence at 12 months. This study found that the rate of sustained 12-month abstinence was $8.4 \%$ (31 participants) in the cytisine group compared with $2.4 \%$ (9 participants) in the placebo group. The 7-day point prevalence for abstinence at the 12 -month follow-up was $13.2 \%$ in the cytisine group and $7.3 \%$ in the placebo group. The relative difference in smoking cessation between cytisine and placebo was higher than previous studies have shown for varenicline and nicotine replacement therapy. However, the absolute difference in the rate of abstinence ( 6 percentage points) was lower than that shown for varenicline and similar to that shown for nicotine replacement therapy. Cytisine resulted in more gastrointestinal adverse events than placebo and rates of discontinuation or dose reduction were similar in both groups.

Cytisine was more effective than placebo for smoking cessation in this single-centre study. The authors believe that combining cytisine with more intensive behavioural support may result in higher absolute quit rates and, by giving a longer regimen, it is possible that efficacy could be improved. However, this study was not large enough for an assessment of uncommon adverse events and it is likely that more studies into the safety and efficacy of cytisine are needed and surveillance for rare side effects undertaken.

West R, Zatonski W, Cedzynska M, et al. Placebo-controlled trial of cytisine for smoking cessation. N Engl J Med 2011;365:1193-200.

\section{Wa'el Osman}

Correspondence to Dr Wa'el Osman, Department of Respiratory Medicine, Dumfries and Galloway Royal Infirmary, Bankend Road, Dumfries DG1 4AP, UK; w.osman@nhs.net

Provenance and peer review Not commissioned; internally peer reviewed.

Published Online First 16 November 2011

Thorax 2012;67:573. doi:10.1136/thoraxjnl-2011-201328 